Ann. Zootech., I970, 19 (3), 347-35I.

NOTE

\title{
FACTEUR FAVORABLE \\ A LA FORMATION DU FOIE GRAS CHEZ L'OIE : LA DISTRIBUTION D'UN RÉGIME ENRICHI EN CHOLINE PENDANT LA PÉRIODE PRÉCÉDANT LE GAVAGE
}

\author{
J.-C. BLUM et B. LECLERCQ \\ Station de Recherches avicoles, \\ Centre de Recherches de Tours, 37 - Nouzilly \\ Institut national de la Recherche agronomique
}

RÉSUMÉ

Au cours du premier essai, l'aliment distribué pendant un mois avant le gavage est supplémenté par 2 p. Ioo de choline. Le foie gras obtenu après un gavage identique pour toutes les oies est plus lourd dans ce lot expérimental ; son poids moyen excède de roo grammes, soit 20 p. 100, celui des témoins.

Le deuxième essai confirme ce résultat. L'influence du traitement est moins marquée chez les animaux jeunes (moins de 5 mois). La distribution du régime enrichi ( 2 p. I oo de choline) pendant un temps très long, depuis la naissance jusqu'au gavage, n'accroît pas les performances. Réduite de moitié ( $\mathrm{p}$. Ioo du régime) la surcharge en facteur lipotrope est sans effet.

L'excès de choline pourrait induire un accroissement du catabolisme, accusant ainsi la déficience au cours du gavage.

\section{INTRODUCTION}

Le gavage de l'Oie pour la production du foie gras est traditionnellement précédé d'une période dite de " prégavage " destinée à préparer l'organisme au gavage et favoriser ainsi le meilleur développement de la stéatose hépatique. A notre connaissance, l'influence sur les performances des conditions nutritionnelles imposées au cours de cette période n'a fait l'objet d'aucune recherche scientifique rigoureuse.

Pour aborder cette étude, nous avons choisi de faire varier l'apport alimentaire de choline pendant le "prégavage ". On sait, en effet, que le foie gras de l'Oie résulte à la fois d'une hyperactivité de l'organe orientée vers la lipogenèse et d'une défaillance du système assurant le transport 
des lipides hors du foie (LECLERCQ et al., i968). La défaillance du système de transport incombe dans une certaine mesure à la pauvreté en choline du régime pendant le gavage. Nous cherchons à accentuer cette déficience. Pour ce faire, nous réalisons, avant le gavage, une surcharge alimentaire de choline en espérant que les enzymes du catabolisme mis en place à cette occasion continueront de fonctionner durant le gavage, exagérant ainsi la carence en facteur lipotrope. Une observation de NESHEIM et al. (I967) fournit des arguments favorables à cette hypothèse : chez la Poule, la dégénérescence graisseuse du foie est plus fréquente en début de ponte, lorsque le régime distribué à la fin de la croissance était riche en choline.

\section{MATÉRIEL ETT MÉTHODES}

Toutes les oies sont de la race Grise des Landes; elles sont élevées au Domaine expérimental d'Artiguères selon les méthodes habituelles (MoNACHON, I966).

\section{Préparation au gavage}

Le premier essai est réalisé en 1968. Les oies âgées de 5 mois sont groupées en 2 lots de 28 sujets. Le lot témoin reçoit le régime de prégavage habituel $(60 \mathrm{p}$. Ioo de maîs, $20 \mathrm{p}$. Ioo de tourteau d'arachide et $20 \mathrm{p}$. Ioo de farine de viande). Le lot expérimental est nourri avec le même régime supplémenté par 2 p. roo de choline. Dans les deux lots, les oies disposent de fourrage vert; l'alimentation est ad libitum. La préparation au gavage est terminée après un mois de régime.

Le deuxième essai est effectué un an plus tard en 1969. On compare alors les effets de 4 traitements :

Témoin : Aucune supplémentation en choline.

$C_{1}$ court: Addition de I p. Ioo de choline à l'aliment consommé pendant I mois avant le gavage.

$\mathrm{C}_{1}$ long : Addition de $\mathrm{I}$ p. Ioo de choline aux aliments consommés depuis l'éclosion jusqu'au gavage.

$\mathrm{C}_{2}$ iong : Addition de 2 p. 100 de choline aux aliments consommés depuis l'éclosion jusqu'au gavage.

Ces 4 traitements sont appliqués à des oies qui sont gavées à un âge variable : 3,4 et 6,5 mois. Pour chaque âge et chaque traitement, on dispose de 20 individus.

\section{Gavage}

Les traitements subis pendant la préparation au gavage définissent les lots. Le régime et la méthode de gavage sont identiques pour toutes les oies. L'aliment est constitué par un mélange de maïs (95 p. 100) et saindoux (5. p. I0o) salé et cuit à la vapeur. Chaque animal ingère 3 repas de 200 grammes de produit sec par jour. Après 3 semaines de gavage, les oies sont sacrifiées; on détermine le poids et la qualité du foie gras.

\section{RÉSULTATS}

Dans le tableau I, nous indiquons les résultats du premier essai. On remarque tout d'abord l'extrême variabilité du poids des foies gras (coefficient de variation voisin de $30 \mathrm{p}$. Ioo). Malgré cette variabilité, on constate que la surcharge en choline permet d'obtenir des foies significativement plus lourds, d'un poids moyen supérieur de roo grammes à celui des témoins. On note également un accroissement de la qualité (plus de foies extra) qui est inattendu, étant donné que la corrélation entre qualité et poids du foie gras est le plus souvent négative. 
Les résultats du second essai sont rassemblés dans le tableau 2. On peut constater que l'âge des animaux a une influence très significative sur les résultats de gavage : les foies sont plus petits chez les animaux jeunes. Par ailleurs, la surcharge en choline la plus forte (2 p. Ioo) permet cette fois encore d'obtenir des foies gras plus lourds. La différence avec les témoins est faible lorsque les oies gavées sont jeunes : à l'âge de 3 et 4 mois, on note seulement une tendance des foies à être plus gros ; le seuil de la signification ( 5 p. Ioo) est atteint chez les sujets âgés de 6,5 mois. La dose de I p. roo de choline est sans effet, qu'elle soit appliquée un mois avant le gavage ou pendant un temps plus long depuis la naissance.

TABIEAU I

Poids et qualité des foies gras obtenus au cours du premier essai

\begin{tabular}{|c|c|c|}
\hline & $\begin{array}{l}\text { Lot témoin } \\
\text { (prégavage normal) }\end{array}$ & $\begin{array}{l}\text { Lot expérimental } \\
(2 \text { p. } 100 \text { de choline } \\
\text { pendant } 1 \text { uois } \\
\text { avant le gavage) }\end{array}$ \\
\hline Nombre d'oies . ........... & 28 & 25 \\
\hline $\begin{array}{l}\text { Poids moyen du foie }(\mathrm{g}) \ldots \ldots \\
\text { et écart-type } \ldots \ldots \ldots \ldots\end{array}$ & $\begin{array}{l}5 / 11,8 \\
165,9\end{array}$ & $\begin{array}{l}642,2 \\
190,8\end{array}$ \\
\hline $\begin{array}{c}\text { Classification } \\
\text { des foies }\end{array}\left\{\begin{array}{l}\text { Extra } \ldots \ldots \\
\text { ge choix } \ldots \ldots \\
\text { Purée } \ldots \ldots\end{array}\right.$ & $\begin{array}{r}18 \\
4 \\
6\end{array}$ & $\begin{array}{r}21 \\
3 \\
3\end{array}$ \\
\hline
\end{tabular}

Analyse statistique des poids de foies : $\mathrm{I}=\{, \geq 0$ significatif au seuil de 5 p. 100

\section{TABLEAU 2}

Poids moyen des foies gras (en grammes) selon l'âge et la préparation des oies gavées au cours du deuxième essai

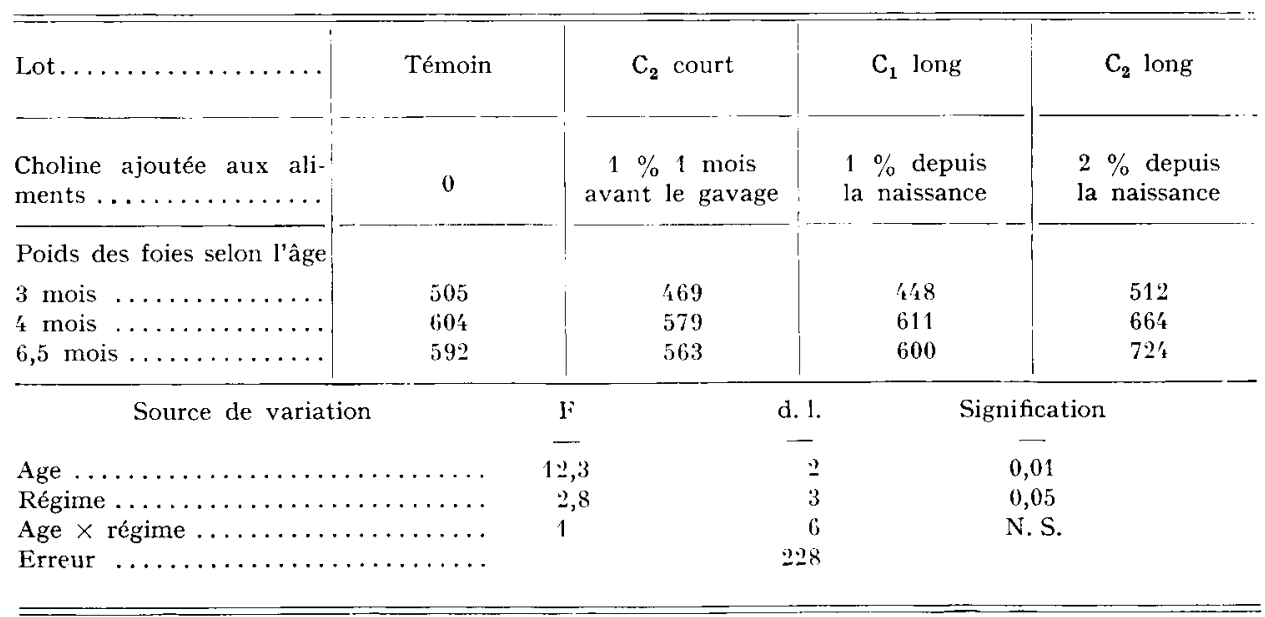




\title{
DISCUSSION
}

L'aptitude à la production du foie gras varie d'un individu à l'autre. L'importance de la variabilité nécessite l'emploi d'effectifs importants et la répétition des essais. Elle nous incite à la prudence dans l'interprétation des résultats. Cependant, nous pouvons affirmer qu'une surcharge en choline avant le gavage favorise le développement de la stéatose hépatique, le seuil de la signification étant atteint au cours des deux expériences effectuées à I an d'intervalle.

Le mécanisme responsable de cette influence de la choline demeure incertain. La déficience peut être accrue pendant le gavage, soit par suite d'une réduction de l'anabolisme, soit parce que le catabolisme est augmenté. La première hypothèse suppose une influence prépondérante du très jeune âge, période pendant laquelle les processus de synthèse sont mis en place, chez le Poussin tout au moins (NoRVell et NESHEIm, I969). Or, nous constatons que les animaux âgés sont plus sensibles et de toute façon que le dernier mois avant gavage entre seul en ligne de compte. Plutôt qu'une suppression de l'anabolisme, il paraît vraisemblable d'envisager une dégradation durablement accrue, véritable adaptation apparaissant seulement lorsque l'ingestion de facteur lipotrope est considérable. Du reste, les enzymes du catabolisme jouent un rôle incontestable dans l'apparition de la carence en choline (Griffith et $\mathrm{NYC}$, I954). Lorsque le régime est déficient, le développement de la stéatose hépatique dépend de l'activité de la choline oxydase; cette activité est forte dans les espèces sensibles, faible ou nulle dans les espèces insensibles (Griffith et Nyc, 1954). Il reste à savoir dans quelle mesure un excès de choline peut entraîner une adaptation enzymatique durable tendant à accroître le catabolisme. La mesure des activités enzymatiques au niveau du foie, en particulier de la choline oxydase, devrait apporter une réponse à ce problème.

En tout cas, l'augmentation de poids des foies gras obtenus est loin d'être négligeable. La nécessité d'un apport massif est compensée par la brièveté de la période d'adaptation. Il reste cependant à préciser la dose et la durée d'administration les moins onéreuses pour une performance maximum.

Reçu pour publication en septembre 1970.

\section{SUMMARY}

\author{
FATTY LIVER DEVELOPMENT IN THE GOOSE : FAVOURABLE \\ INFLUENCE OF FEEDING CHOLINE ENRICHED DIETS \\ DURING THE PRE-CRAMMING PERIOD
}

During the first trial, the food rations allotted before the cramming were supplemented by 2 p. Ioo of choline. All the geese were crammed in an identical way, but the livers obtained were heavier in the experimental group; their mean weight being $100 \mathrm{~g}$ higher (20 p. 100) than that of the controls.

The second trial confirms the results. The influence of the treatment is less conspicuous in the animals younger than 5 months. If the enriched diet $(2 \mathrm{p}$. Ioo choline) is fed during a very long time, $i$. e. since birth and until the cramming period, the performances are not increased. Reduced by half (I p. roo of the diet), the overload of the lipotropic factor is without effect.

Excess of choline could induce an increase of the catabolism, thus accentuating the deficiency during the cramming. 


\section{RÉFÉRENCES BIBIIOGRAPHIQUES}

Griffith W. H., Nyc J. F., I954. Choline, IV. Biochemical systems in The vitamins, vol. II, 15-45, edit. Sebrell and Harris, Academic Press, New York.

LeclercQ B., Durand G., Delpech P., Blum J.-C., I968. Note préliminaire sur l'évolution des constituants biochimiques du foie au cours du gavage de l'Oie. Ann. Biol. anim. Bioch. Biophys., 8, 549-556.

Monachon G., Ig66. Contribution du Domaine expérimental d'Artiguère à l'amélioration de l'élevage de l'Oie. XIII' Congr. Mond. Avic., Kiev.

Nesheim M. C., Leballos E., Leach R. M., Norvell M. J., I967. The effect of dietary choline on growth of pullets and subsequent effects on egg production and liver lipid. Poult. Sci., 46, I299.

Norvell M. J., Nesheim M. C., I969. Studies on rate of choline synthesis in chicks and laying hens. Proc. Cornell Nutr. Conf., 3I-34. 